\title{
A moderate-carbohydrate diet with plant protein is inversely associated with cardiovascular risk factors: the Korea National Health and Nutrition Examination Survey 2013-2017
}

Kyungho $\mathrm{Ha}^{1}$, Kisun $\mathrm{Nam}^{2}$ and YoonJu Song ${ }^{3 *}$

\begin{abstract}
Background: Because a moderate-carbohydrate diet reportedly has minimal risks, the substitution of carbohydrate for protein has been emphasized. Few studies have explored the effect of moderate-carbohydrate diets with higher protein intake in Asians, who typically consume a high-carbohydrate low-fat diet. Therefore, this study evaluated the associations of moderate- versus high- carbohydrate diets with cardiovascular risk factors among Korean adults by protein source.
\end{abstract}

Methods: This study included 7965 adults (3196 men, 4769 women) aged $\geq 19$ years who participated in the 2013-2017 Korea National Health and Nutrition Examination Survey. Dietary intake was assessed by a 24-h recall method and four types of diet were defined: a moderate-carbohydrate diet with plant protein (MCP) or animal protein (MCA) and a high-carbohydrate diet with plant protein (HCP) or animal protein (HCA).

Results: Compared with the MCP group, men in the other three groups had significantly higher odds ratios (ORs) for elevated total cholesterol, reduced high-density lipoprotein (HDL)-cholesterol, and metabolic syndrome. Among women, only the HCP group had an increased OR for reduced HDL-cholesterol, compared with the MCP group. Similar associations were observed in younger adults (19-49 years). In addition, younger adults in the MCA group exhibited higher ORs for elevated triglycerides in men and elevated total cholesterol in women, compared with those in the MCP group.

Conclusions: A moderate-carbohydrate diet with a high intake of plant protein was inversely associated with cardiovascular risk factors, especially among younger Korean adults. Further intervention studies are required to confirm this relationship and develop the optimal diet for cardiovascular health in the Korean population.

Keywords: Moderate carbohydrate diet, Plant protein, Dyslipidemia, Metabolic syndrome, Korea

\footnotetext{
* Correspondence: yjsong@catholic.ac.kr

${ }^{3}$ Major of Food and Nutrition, The Catholic University of Korea, 43 Jibong-ro, Wonmi-gu, Bucheon-si, Gyeonggi-do 14662, Republic of Korea

Full list of author information is available at the end of the article
}

(C) The Author(s). 2020 Open Access This article is licensed under a Creative Commons Attribution 4.0 International License, which permits use, sharing, adaptation, distribution and reproduction in any medium or format, as long as you give appropriate credit to the original author(s) and the source, provide a link to the Creative Commons licence, and indicate if changes were made. The images or other third party material in this article are included in the article's Creative Commons licence, unless indicated otherwise in a credit line to the material. If material is not included in the article's Creative Commons licence and your intended use is not permitted by statutory regulation or exceeds the permitted use, you will need to obtain permission directly from the copyright holder. To view a copy of this licence, visit http://creativecommons.org/licenses/by/4.0/ The Creative Commons Public Domain Dedication waiver (http://creativecommons.org/publicdomain/zero/1.0/) applies to the data made available in this article, unless otherwise stated in a credit line to the data. 


\section{Background}

In recent decades, research interest in a lowcarbohydrate diet versus a low-fat diet has increased due to its health effects. A low-fat diet has been long emphasized to prevent and manage cardiovascular diseases, while a low-carbohydrate diet has an effect on weight loss comparable to a low-fat diet [1, 2]. In terms of cardiovascular risk factors, a low-carbohydrate diet has been associated with a decreased triglyceride level and increased high-density lipoprotein (HDL)-cholesterol level in meta-analyses of randomized controlled trials $[3,4]$.

There is no universal definition of a low-carbohydrate diet and several types have been formulated [2, 5]. In addition, carbohydrate intake differs among countries and a low-carbohydrate intake can be defined differently by population. For example, the average intake of carbohydrate is approximately $45-50 \%$ of energy among adults from the United States (US), United Kingdom, and Canada [6-8], compared with $\sim 60 \%$ among adults from Korea and Japan [9, 10]. Nevertheless, a lowcarbohydrate diet is typically regarded as a carbohydrate intake of less than $45 \%$ [11] or $40 \%$ [5] of the total energy.

A recent prospective study reported a minimal risk of mortality for a moderate carbohydrate intake (50-55\% of energy), while high- and low-carbohydrate diets were related to increased mortality [12]. Additionally, substitution of carbohydrate for animal protein and fat in a low-carbohydrate diet was associated with an increased risk of mortality, compared with substitution of carbohydrate for plant protein and fat [12].

High-protein diets have been a focus with regard to replacing carbohydrate or fat in the diet $[13,14]$. Noakes et al. [13] reported that a high-protein diet was associated with a greater reduction in fat mass and body weight compared with conventional high-carbohydrate low-fat diets, undertaken for 12 weeks, among subjects with an elevated serum triacylglycerol level at baseline. Morenga et al. [14] reported that a relatively highprotein high-fiber diet for 10 weeks improved body composition and metabolic risk factors, compared with a standard low-fat high-carbohydrate diet in overweight women.

The source of dietary protein (plant or animal) is important for cardiometabolic health $[15,16]$. According to a recent systematic review, soy protein may reduce the risk of cardiovascular diseases such as hypercholesterolemia and hypertension [16]. Similarly, recent longitudinal studies showed that high intake of protein from nuts and seeds in US adults was associated with decreased mortality from cardiovascular disease [17]; moreover, animal protein intake in Australian adults was associated with an increased incidence of metabolic syndrome, whereas plant protein intake showed the opposite association [18]. However, few studies have explored the effect of moderate-carbohydrate diets according to protein source in Asian populations, who typically consume a high-carbohydrate low-fat diet. Thus, this study evaluated associations of cardiovascular risk factors with moderate- and high-carbohydrate diets according to protein source among Korean adults.

\section{Methods}

\section{Study design and participants}

This study used cross-sectional data from the 20132017 Korea National Health and Nutrition Examination Survey (KNHANES). KNHANES is a nationwide survey conducted by the Korea Centers for Disease Control \& Prevention (KCDC) to evaluate the health and nutritional status of Koreans; it comprises a health examination, health interview, and nutrition survey. To select a nationally representative sample of the Korean population, approximately 10,000 non-institutionalized individuals aged $\geq 1$ years are recruited each year, in accordance with a complex multi-stage clustered probability design. The details of KNHANES are available elsewhere [19].

Among 27,220 adults aged 19 years or older who participated in a 24-h dietary recall survey, those who did not have information regarding anthropometric and biochemical measurements such as waist circumference, blood pressure, blood glucose, and blood lipid levels $(n=3992)$; those who had been diagnosed with or were taking medication for diabetes, hypertension, or dyslipidemia $(n=8128)$; those who had been diagnosed with stroke, myocardial infarction, or angina $(n=232)$, those women who were pregnant or lactating $(n=225)$; and those who reported an implausible energy intake $(<500$ or $>5000 \mathrm{kcal} /$ day $)(n=287)$ were excluded. A total of 14,356 participants were eligible for this study.

\section{Assessment of dietary intake}

Energy and macronutrient intakes, including intake of dietary fiber, were assessed using a 1-day 24-h dietary recall method. A trained staff member visited the household of the participant and measured all foods and beverages consumed by the participant within $24 \mathrm{~h}$ before the day preceding the survey. Carbohydrate, protein, and fat intakes were estimated as absolute intake (g/day) and percentage of total energy (\% of total energy). Protein and fat intakes were divided into plant or animal protein and fat according to food sources. The sources of plant protein and fat were plant foods, including beans, nuts, grains, and vegetables; the sources of animal protein and fat were animal foods including meat (beef, pork, poultry, and other), fish (excluding seaweeds), eggs, and milk and dairy products (yogurt, cheese, and other). Based on the 2015 Dietary Reference Intakes for Koreans (KDRIs) [20], energy intake was compared with the 
estimated energy requirement (EER) to evaluate the adequacy of energy intake.

Food items consumed were categorized into grains (300 kcal/serving); meat, fish, eggs, and beans (MFEB) (100 kcal/serving); vegetables ( $15 \mathrm{kcal} /$ serving); fruits (50 $\mathrm{kcal} /$ serving); and milk and dairy products (125 kcal/ serving). Food group consumption was calculated as the number of servings per day. Food group consumption was evaluated compared with the recommended number of servings for each food group based on the recommended dietary pattern by life stage of the KDRIs [20].

\section{Definitions of moderate- and high-carbohydrate diets}

A moderate-carbohydrate diet with plant protein intake was defined based on the 211 Diet, a balanced meal plan developed by Pulmuone (Seoul, Korea). The 211 Diet emphasizes the selection of protein foods with low saturated fat, fresh vegetables, and whole grains to prevent excessive carbohydrate consumption. At the nutrient level, the 211 diet recommends consuming $50 \%$ of daily energy from carbohydrates and higher plant protein, compared with animal protein, which can be defined using the ratio of plant protein to animal protein. Considering the overall high carbohydrate intake in Korean adults [9], moderate- and high-carbohydrate diets were defined as $50-60 \%$ and $\geq 70 \%$, respectively. Thus, four types of diets were defined according to carbohydrate and protein intake (Table 1): moderate-carbohydrate diet with plant protein $(\mathrm{MCP})$, moderate-carbohydrate diet with animal protein (MCA), high-carbohydrate diet with plant protein (HCP), and high-carbohydrate diet with animal protein (HCA). If participants did not consume any animal protein, the ratio of plant to animal protein was regarded as $\geq 1$. After exclusion of those who did not satisfy the criteria of these moderate- and highcarbohydrate diets, 7965 participants (3196 men and 4769 women) were selected for the final analysis.

\section{Measurement of anthropometric and biochemical variables}

Anthropometric variables (weight, height, and waist circumference), blood pressure, and biochemical variables (total cholesterol, HDL-cholesterol, triglycerides, and fasting glucose levels) were measured at a mobile examination center using standardized procedures and calibrated equipment. Body mass index (BMI) was calculated based on weight and height $\left(\mathrm{kg} / \mathrm{m}^{2}\right)$. Systolic and diastolic blood pressure (SBP and DBP) were measured three times and the average of the second and third readings was used. Blood indices of participants who fasted for at least $8 \mathrm{~h}$ were used. Low-density lipoprotein (LDL)-cholesterol was calculated in accordance with the Friedewald formula only for participants whose triglyceride level was $<400 \mathrm{mg} / \mathrm{dL}$ [21]. The details of the laboratory procedures are available elsewhere [22].

\section{Cardiovascular risk factors}

Cardiovascular risk factors assessed in this study were dyslipidemia, metabolic syndrome, hypertension, and type 2 diabetes. Dyslipidemia, which comprises several lipid abnormalities, was diagnosed as follows in accordance with the Korean Guidelines for the Management of Dyslipidemia (4th edition) [23]: 1) elevated total cholesterol ( $\geq 240 \mathrm{mg} / \mathrm{dL}$ ), 2) elevated triglycerides ( $\geq 200 \mathrm{mg} /$ $\mathrm{dL})$, 3) elevated LDL-cholesterol ( $\geq 160 \mathrm{mg} / \mathrm{dL})$, and 4$)$ reduced HDL-choletserol $(<40 \mathrm{mg} / \mathrm{dL})$. Based on the National Cholesterol Education Program Adult Treatment Panel III criteria [24], with the exception of waist circumference [25], metabolic syndrome was defined as the presence of three or more of the following metabolic abnormalities: 1) increased waist circumference $(\geq 90 \mathrm{~cm}$ for men and $\geq 85 \mathrm{~cm}$ for women), 2) elevated blood pressure (SBP $\geq 130 \mathrm{mmHg}$ or $\mathrm{DBP} \geq 85 \mathrm{mmHg}$ ), 3) reduced HDL-cholesterol $(<40 \mathrm{mg} / \mathrm{dL}$ for men and $<50 \mathrm{mg} / \mathrm{dL}$ for women), 4) elevated triglycerides ( $\geq 150 \mathrm{mg} / \mathrm{dL}$ ), and 5) elevated fasting glucose $(\geq 100 \mathrm{mg} / \mathrm{dL})$. Hypertension was defined as an SBP of $\geq 140 \mathrm{mmHg}$ or a DBP of $\geq 90$ $\mathrm{mmHg}$ and type 2 diabetes as a fasting glucose level of $\geq 126 \mathrm{mg} / \mathrm{dL}$.

\section{Assessment of confounders}

Sociodemographic characteristics (e.g., age, sex, education level, and household income) and health behaviors (e.g., physical activity, alcohol consumption, and smoking) were assessed in a health interview at the mobile examination center and were included as potential confounders. Education level was classified into middle school or lower, high school, or college or higher. Household income was divided into lowest, lower middle, upper middle, or highest quartile of monthly household income. Physical activity was defined as "yes," if

Table 1 Moderate- and high-carbohydrate diets by protein source

\begin{tabular}{lll}
\hline Diet & Carbohydrate intake (\% of energy) & Ratio of plant to animal protein \\
\hline Moderate-carbohydrate diet with plant protein & $50-60 \%$ & $\geq 1$ \\
Moderate-carbohydrate diet with animal protein & $50-60 \%$ & $<1$ \\
High-carbohydrate diet with plant protein & $\geq 70 \%$ & $\geq 1$ \\
High-carbohydrate diet with animal protein & $\geq 70 \%$ & $<1$ \\
\hline
\end{tabular}


Table 2 General characteristics of the participants according to moderate- and high-carbohydrate diets, stratified by protein source

\begin{tabular}{|c|c|c|c|c|c|}
\hline$\overline{n,(\%)^{a}}$ & $\begin{array}{l}\text { Moderate carbohydrate diet } \\
\text { with plant protein }(\mathrm{MCP})^{\mathbf{b}} \\
(\boldsymbol{n}=981)\end{array}$ & $\begin{array}{l}\text { Moderate carbohydrate diet } \\
\text { with animal protein }(\text { MCA })^{\mathbf{b}} \\
(\boldsymbol{n}=2209)\end{array}$ & $\begin{array}{l}\text { High carbohydrate diet } \\
\text { with plant protein }(\mathrm{HCP})^{\mathbf{b}} \\
(\boldsymbol{n}=4283)\end{array}$ & $\begin{array}{l}\text { High carbohydrate diet } \\
\text { with animal protein (HCA) } \\
(\boldsymbol{n}=492)\end{array}$ & $\boldsymbol{P}$ value \\
\hline Sex & & & & & $<0.0001$ \\
\hline Male & $392(50.8)$ & $992(54.3)$ & $1610(45.1)$ & $202(50.2)$ & \\
\hline Female & 589 (49.2) & $1217(45.7)$ & $2673(54.9)$ & $290(49.8)$ & \\
\hline $\begin{array}{l}\text { Age group } \\
\text { (years) }\end{array}$ & & & & & $<0.0001$ \\
\hline $19-29$ & $230(32.1)$ & $541(32.7)$ & $328(13.2)$ & $85(24.5)$ & \\
\hline $30-49$ & 517 (49.6) & $1221(52.1)$ & 1441 (39.3) & $201(42.9)$ & \\
\hline $50-64$ & 189 (15.6) & $354(13.0)$ & 1458 (31.8) & $140(24.1)$ & \\
\hline$\geq 65$ & $45(2.7)$ & $93(2.3)$ & $1056(15.7)$ & $66(8.5)$ & \\
\hline Survey period & & & & & $<0.0001$ \\
\hline 2013-2015 & $552(57.5)$ & 1204 (55.6) & $2649(62.8)$ & $277(58.2)$ & \\
\hline 2016-2017 & $429(42.5)$ & $1005(44.4)$ & $1634(37.2)$ & $215(41.8)$ & \\
\hline Education & & & & & $<0.0001$ \\
\hline $\begin{array}{l}\text { Middle } \\
\text { school or } \\
\text { lower }\end{array}$ & $101(8.2)$ & $186(7.0)$ & 1595 (28.9) & 107 (16.2) & \\
\hline High school & $362(40.1)$ & 864 (41.5) & $1364(36.8)$ & 177 (39.9) & \\
\hline $\begin{array}{l}\text { College or } \\
\text { higher }\end{array}$ & $502(51.7)$ & $1115(51.4)$ & $1227(34.3)$ & $195(43.9)$ & \\
\hline $\begin{array}{l}\text { Household } \\
\text { income }\end{array}$ & & & & & $<0.0001$ \\
\hline Lowest & $67(7.0)$ & $163(7.4)$ & 889 (16.8) & $67(11.9)$ & \\
\hline $\begin{array}{l}\text { Lower } \\
\text { middle }\end{array}$ & $233(23.4)$ & 478 (21.6) & 1164 (26.8) & $117(22.3)$ & \\
\hline $\begin{array}{l}\text { Upper } \\
\text { middle }\end{array}$ & $335(34.8)$ & $757(34.6)$ & 1131 (28.6) & $142(29.6)$ & \\
\hline Highest & $343(34.7)$ & $807(36.4)$ & $1089(27.8)$ & $164(36.3)$ & \\
\hline $\begin{array}{l}\text { Physical } \\
\text { activity, yes }^{c}\end{array}$ & $529(58.7)$ & $1106(54.6)$ & $1811(46.9)$ & $243(53.5)$ & $<0.0001$ \\
\hline $\begin{array}{l}\text { Alcohol } \\
\text { consumption }^{d}\end{array}$ & & & & & $<0.0001$ \\
\hline None & $356(32.3)$ & $745(30.9)$ & $2279(48.6)$ & $210(37.5)$ & \\
\hline Moderate & 499 (53.5) & $1120(52.5)$ & $1674(43.6)$ & $224(49.4)$ & \\
\hline High & $123(14.2)$ & 334 (16.6) & $282(7.9)$ & $54(13.1)$ & \\
\hline Smoking ${ }^{e}$ & & & & & $<0.0001$ \\
\hline Never & $638(59.7)$ & $1406(60.1)$ & $2897(64.2)$ & 311 (59.3) & \\
\hline Former & $141(14.8)$ & $321(14.4)$ & $726(17.7)$ & $81(16.9)$ & \\
\hline Current & 199 (25.6) & $472(25.4)$ & $606(18.1)$ & $95(23.8)$ & \\
\hline
\end{tabular}

${ }^{a}$ Values are presented as the weighted percentages of the participants using the complex sampling design parameters of KNHANES

${ }^{\mathrm{b}} \mathrm{MCP}=$ carbohydrate intake $50-60 \%$ of energy + plant $/$ animal protein $\geq 1 ; \mathrm{MCA}=$ carbohydrate intake $50-60 \%$ of energy + plant $/$ animal protein $<1 ; \mathrm{HCP}=$ carbohydrate intake $\geq 70 \%$ of energy + plant/animal protein $\geq 1 ; \mathrm{HCA}=$ carbohydrate intake $\geq 70 \%$ of energy + plant/animal protein $<1$

" Physical activity: "yes", performed vigorous-intensity activities for at least $75 \mathrm{~min}$, or moderate-intensity activities for at least $150 \mathrm{~min}$, or an equivalent combination of moderate- and vigorous-intensity activity during a typical week

"Alcohol consumption: "none", no consumption of any type of alcoholic beverage or drank less than once per month over the past year, "moderate", consumption of alcoholic beverages more than once per month over the past year, "high", consumption of more than seven glasses of alcoholic beverages for men and five glasses of alcoholic beverages for women per occasion more than two times per week

e Smoking: "never", never smoked cigarettes of smoked $<100$ cigarettes in their lifetime, "former", smoked $\geq 100$ cigarettes in their lifetime but currently not smoking, "current", smoked $\geq 100$ cigarettes in their lifetime and currently smoking 
participants performed vigorous-intensity activities for at least $75 \mathrm{~min}$, or moderate-intensity activities for at least $150 \mathrm{~min}$, or an equivalent combination of moderate- and vigorous-intensity activity during a typical week. Alcohol consumption was categorized as none (no consumption of any type of alcoholic beverage or less than once per month over the past year), moderate (consumption of alcoholic beverages more than once per month over the past year), and high (consumption of more than seven glasses of alcoholic beverages for men and five glasses of alcoholic beverages for women per occasion more than two times per week). Smoking status was classified as never (never smoked cigarettes or smoked $<100$ cigarettes in their lifetime), former (smoked $\geq 100$ cigarettes in their lifetime but currently not smoking), and current (smoked $\geq 100$ cigarettes in their lifetime and currently smoking). Survey period, BMI, and total energy intake were also considered as confounding variables.

\section{Statistical analysis}

Statistical analysis was carried out using SAS software (ver. 9.4; SAS Institute, Cary, NC, USA). The complex sampling design parameters of KNHANES such as strata, cluster, and weight were used in the PROC SURVEY procedures to represent the Korean population. All $p$-values were two sided and a $p$ value $<0.05$ was considered indicative of statistical significance.

Continuous variables are presented as means \pm standard errors (SEs) and categorical variables are presented as numbers (percentages). Continuous variables were log-transformed to normalize their distributions; these values were used for statistical tests. Differences in general characteristics among the diets were examined using the chi-square test. Adjusted mean values of nutrient and food group intakes were calculated using a general linear model after adjustment for confounders. Multiple logistic regression analysis was performed to estimate the odds ratios (ORs) and 95\% confidence intervals (CIs) of cardiovascular risk factors according to sex with a moderate-carbohydrate diet with plant protein (MCP) set as a reference after adjustment for confounders. This analysis was further stratified according to age group (19-49 and $\geq 50$ years) for each sex. In the age-stratified analysis, type 2 diabetes was excluded due to the small number of cases.

Table 3 Energy and macronutrient intakes according to moderate- and high-carbohydrate diets, stratified by protein source

\begin{tabular}{|c|c|c|c|c|c|}
\hline & $\begin{array}{l}\text { Moderate carbohydrate } \\
\text { diet with plant protein } \\
(\mathrm{MCP})^{\mathbf{a}} \\
(\boldsymbol{n}=981)\end{array}$ & $\begin{array}{l}\text { Moderate carbohydrate } \\
\text { diet with animal protein } \\
(M C A)^{\mathbf{a}} \\
(\boldsymbol{n}=2209)\end{array}$ & $\begin{array}{l}\text { High carbohydrate diet } \\
\text { with plant protein }(\mathrm{HCP})^{\mathbf{a}} \\
(\boldsymbol{n}=4283)\end{array}$ & $\begin{array}{l}\text { High carbohydrate diet } \\
\text { with animal protein }(\mathrm{HCA})^{\mathrm{a}} \\
(\boldsymbol{n}=492)\end{array}$ & $\boldsymbol{P}$ value $^{\mathbf{b}}$ \\
\hline Energy (kcal) & $2170.7 \pm 29.9^{c}$ & $2235.3 \pm 22.1$ & $1871.6 \pm 18.2$ & $1783.2 \pm 38.2$ & $<0.0001$ \\
\hline Energy (\% of EER) & $98.4 \pm 1.3$ & $101.1 \pm 0.9$ & $85.9 \pm 0.8$ & $82.2 \pm 1.7$ & $<0.0001$ \\
\hline Carbohydrate (g) & $252.0 \pm 1.8$ & $238.2 \pm 1.5$ & $348.1 \pm 1.5$ & $329.6 \pm 2.8$ & $<0.0001$ \\
\hline Protein (g) & $64.8 \pm 0.7$ & $83.5 \pm 0.7$ & $55.2 \pm 0.4$ & $68.1 \pm 0.7$ & $<0.0001$ \\
\hline Plant protein & $42.3 \pm 0.5$ & $28.2 \pm 0.3$ & $38.4 \pm 0.3$ & $30.0 \pm 0.4$ & $<0.0001$ \\
\hline Animal protein & $22.4 \pm 0.5$ & $55.2 \pm 0.6$ & $16.7 \pm 0.3$ & $37.9 \pm 0.5$ & $<0.0001$ \\
\hline Fat (g) & $62.1 \pm 0.6$ & $53.8 \pm 0.4$ & $24.7 \pm 0.3$ & $23.9 \pm 0.6$ & $<0.0001$ \\
\hline Plant fat & $43.5 \pm 0.7$ & $23.6 \pm 0.4$ & $16.7 \pm 0.3$ & $12.1 \pm 0.5$ & $<0.0001$ \\
\hline Animal fat & $18.6 \pm 0.5$ & $30.1 \pm 0.4$ & $8.0 \pm 0.2$ & $11.9 \pm 0.4$ & $<0.0001$ \\
\hline Dietary fiber (g) & $23.8 \pm 0.5$ & $19.7 \pm 0.3$ & $24.9 \pm 0.3$ & $21.6 \pm 0.6$ & $<0.0001$ \\
\hline \multicolumn{6}{|l|}{ Energy from (\%) } \\
\hline Carbohydrate & $56.9 \pm 0.1$ & $56.1 \pm 0.1$ & $76.3 \pm 0.1$ & $73.5 \pm 0.1$ & $<0.0001$ \\
\hline Protein & $14.2 \pm 0.1$ & $18.1 \pm 0.1$ & $11.9 \pm 0.1$ & $15.1 \pm 0.1$ & $<0.0001$ \\
\hline Fat & $28.9 \pm 0.2$ & $25.8 \pm 0.1$ & $11.8 \pm 0.1$ & $11.4 \pm 0.2$ & $<0.0001$ \\
\hline SFA & $8.5 \pm 0.1$ & $7.7 \pm 0.1$ & $3.5 \pm 0.0$ & $3.4 \pm 0.1$ & $<0.0001$ \\
\hline MUFA & $9.3 \pm 0.1$ & $8.4 \pm 0.1$ & $3.3 \pm 0.0$ & $3.2 \pm 0.1$ & $<0.0001$ \\
\hline PUFA & $7.4 \pm 0.1$ & $6.1 \pm 0.1$ & $3.1 \pm 0.0$ & $2.9 \pm 0.1$ & $<0.0001$ \\
\hline
\end{tabular}

Abbreviations: EER Estimated energy requirement, MUFA Monounsaturated fatty acids, PUFA Polyunsaturated fatty acids, SFA Saturated fatty acids

${ }^{\mathrm{a}} \mathrm{MCP}=$ carbohydrate intake $50-60 \%$ of energy + plant/animal protein $\geq 1 ; \mathrm{MCA}=$ carbohydrate intake $50-60 \%$ of energy + plant/animal protein $<1 ; \mathrm{HCP}=$

carbohydrate intake $\geq 70 \%$ of energy + plant/animal protein $\geq 1 ; \mathrm{HCA}=$ carbohydrate intake $\geq 70 \%$ of energy + plant/animal protein $<1$

${ }^{\mathrm{b}}$ Log-transformed values were used for statistical tests

'Values are presented as means \pm SEs after adjustment for age, sex, body mass index, education, household income, physical activity, smoking, survey period,

alcohol consumption, and total energy intake (except the model of energy intake) 


\section{Results}

\section{General characteristics of the participants}

The distribution of sociodemographic characteristics and health behaviors differed among the diets (Table 2). Participants with a high-carbohydrate diet ( $\geq 70 \%$ of energy) were older than those with a moderate-carbohydrate diet (50-60\% of energy) $(p<0.0001)$. Education level and household income tended to be lower in the high than the moderate carbohydrate diet groups $(p<0.0001$ for all); they were lowest in participants with a highcarbohydrate diet with plant protein (HCP). The proportion of participants who performed physical activity was highest among those with a moderate-carbohydrate diet with plant protein $(\mathrm{MCP})$ and lowest among those with an HCP $(p<0.0001)$. Participants who consumed more animal than plant protein were more likely to drink alcohol frequently, compared with those who consumed more plant than animal protein $(p<0.0001)$. The proportion of current smokers was lowest among participants with an HCP $(p<0.0001)$.

\section{Dietary intakes}

Energy intake was higher in the moderate than the highcarbohydrate diet groups $(p<0.0001)$ (Table 3$)$. However, the energy intake of the moderate-carbohydrate diet groups was at a satisfactory level, compared with the EER. Dietary fiber intake was higher in participants with higher intake of plant protein, compared with participants with higher intake of animal protein $(p<0.0001)$.

The grain intake of the high-carbohydrate diet with plant protein $(\mathrm{HCP})$ group was highest $(p<0.0001)$ and was approximately $115 \%$ of the recommended dietary pattern (Table 4). The MFEB and grain intakes of the moderate-carbohydrate diet with plant protein (MCP) group were 81.2 and $96.0 \%$ of the recommended level, respectively; however, the fruit intake was $52.6 \%$ of the recommended dietary pattern, which was lower than that of the high-carbohydrate diet groups.

\section{Association of moderate- and high-carbohydrate diets with cardiovascular risk factors}

Table 5 presents the multivariable-adjusted ORs and 95\% CIs of cardiovascular risk factors according to sex. In men, the high-carbohydrate diet with plant protein (HCP) and with animal protein (HCA) groups had higher ORs for an elevated total cholesterol level, reduced HDL-cholesterol level, and metabolic syndrome, compared with the moderate-carbohydrate diet with plant protein (MCP) group; HCP was associated with

Table 4 Food group consumption according to moderate- and high-carbohydrate diets, stratified by protein source

\begin{tabular}{|c|c|c|c|c|c|}
\hline & $\begin{array}{l}\text { Moderate carbohydrate } \\
\text { diet with plant protein } \\
(\mathrm{MCP})^{\mathbf{a}} \\
(\boldsymbol{n}=981)\end{array}$ & $\begin{array}{l}\text { Moderate carbohydrate } \\
\text { diet with animal protein } \\
(M C A)^{\mathbf{a}} \\
(\boldsymbol{n}=2209)\end{array}$ & $\begin{array}{l}\text { High carbohydrate } \\
\text { diet with plant protein } \\
(\mathrm{HCP})^{\mathbf{a}} \\
(\boldsymbol{n}=4283)\end{array}$ & $\begin{array}{l}\text { High carbohydrate } \\
\text { diet with animal protein } \\
(\text { HCA })^{\mathbf{a}} \\
(\boldsymbol{n}=492)\end{array}$ & $\boldsymbol{P}$ value $^{\mathbf{b}}$ \\
\hline \multicolumn{6}{|l|}{ No of servings } \\
\hline Grain & $3.3 \pm 0.0^{c}$ & $2.4 \pm 0.0$ & $4.0 \pm 0.0$ & $3.3 \pm 0.1$ & $<0.0001$ \\
\hline MFEB & $3.5 \pm 0.1$ & $5.3 \pm 0.1$ & $1.8 \pm 0.0$ & $2.8 \pm 0.1$ & $<0.0001$ \\
\hline Meat & $1.6 \pm 0.1$ & $3.4 \pm 0.1$ & $0.7 \pm 0.0$ & $1.2 \pm 0.1$ & $<0.0001$ \\
\hline Fish & $0.4 \pm 0.0$ & $0.9 \pm 0.0$ & $0.4 \pm 0.0$ & $1.1 \pm 0.0$ & $<0.0001$ \\
\hline Eggs & $0.4 \pm 0.0$ & $0.5 \pm 0.0$ & $0.3 \pm 0.0$ & $0.3 \pm 0.0$ & $<0.0001$ \\
\hline Beans & $1.1 \pm 0.1$ & $0.5 \pm 0.0$ & $0.4 \pm 0.0$ & $0.3 \pm 0.0$ & $<0.0001$ \\
\hline Vegetables & $6.3 \pm 0.2$ & $6.5 \pm 0.1$ & $6.7 \pm 0.1$ & $6.0 \pm 0.2$ & 0.0102 \\
\hline Fruits & $1.0 \pm 0.1$ & $1.2 \pm 0.1$ & $2.2 \pm 0.1$ & $2.6 \pm 0.2$ & $<0.0001$ \\
\hline Milk and dairy products & $0.5 \pm 0.0$ & $0.6 \pm 0.0$ & $0.3 \pm 0.0$ & $0.5 \pm 0.1$ & $<0.0001$ \\
\hline \multicolumn{6}{|l|}{$\%$ Servings $^{d}$} \\
\hline Grain & $96.0 \pm 1.3$ & $69.6 \pm 0.9$ & $114.6 \pm 0.8$ & $94.9 \pm 1.4$ & $<0.0001$ \\
\hline MFEB & $81.2 \pm 1.8$ & $120.5 \pm 1.1$ & $41.2 \pm 0.7$ & $64.8 \pm 1.2$ & $<0.0001$ \\
\hline Vegetables & $80.3 \pm 2.3$ & $82.2 \pm 1.4$ & $85.0 \pm 1.3$ & $76.7 \pm 2.4$ & 0.0115 \\
\hline Fruits & $52.6 \pm 3.8$ & $61.8 \pm 3.1$ & $102.3 \pm 3.1$ & $121.2 \pm 6.5$ & $<0.0001$ \\
\hline Milk and dairy products & $45.6 \pm 3.7$ & $62.8 \pm 3.0$ & $28.9 \pm 2.0$ & $53.3 \pm 5.8$ & $<0.0001$ \\
\hline
\end{tabular}

Abbreviations: MFEB Meat, fish, eggs, and beans

${ }^{\mathrm{a}} \mathrm{MCP}=$ carbohydrate intake $50-60 \%$ of energy + plant/animal protein $\geq 1 ; \mathrm{MCA}=$ carbohydrate intake $50-60 \%$ of energy + plant $/$ animal protein $<1 ; \mathrm{HCP}=$ carbohydrate intake $\geq 70 \%$ of energy + plant/animal protein $\geq 1 ; \mathrm{HCA}=$ carbohydrate intake $\geq 70 \%$ of energy + plant/animal protein $<1$

${ }^{b}$ Log-transformed values were used for statistical tests

'Values are presented as means \pm SEs after adjustment for age, sex, body mass index, education, household income, physical activity, smoking, survey period, alcohol consumption, and total energy intake

$\mathrm{d}_{\% \text { Servings }}=$ the number of servings consumed $\div$ recommended number of servings based on Dietary Reference Intakes for Koreans $\times 100$ 
Table 5 Multivariable-adjusted odds ratios and 95\% confidence intervals of cardiovascular risk factors according to moderate- and high-carbohydrate diets, stratified by protein source

\begin{tabular}{|c|c|c|c|c|}
\hline Men & $\begin{array}{l}\text { Moderate carbohydrate diet } \\
\text { with plant protein }(\mathrm{MCP})^{\mathrm{a}} \\
(n=392)\end{array}$ & $\begin{array}{l}\text { Moderate carbohydrate diet } \\
\text { with animal protein (MCA) } \\
(n=992)\end{array}$ & $\begin{array}{l}\text { High carbohydrate diet } \\
\text { with plant protein }(\mathrm{HCP})^{\mathrm{a}} \\
(n=1610)\end{array}$ & $\begin{array}{l}\text { High carbohydrate diet } \\
\text { with animal protein (HCA) } \\
(n=202)\end{array}$ \\
\hline \multicolumn{5}{|l|}{ Dyslipidemia } \\
\hline $\begin{array}{l}\text { Elevated total } \\
\text { cholesterol }\end{array}$ & $1.00^{\mathrm{b}}$ & $1.80(1.07-3.04)$ & $1.94(1.15-3.25)$ & $2.11(1.04-4.30)$ \\
\hline $\begin{array}{l}\text { Elevated } \\
\text { triglycerides }\end{array}$ & 1.00 & $0.92(0.65-1.30)$ & $1.05(0.74-1.48)$ & $0.90(0.52-1.55)$ \\
\hline $\begin{array}{l}\text { Elevated LDL- } \\
\text { cholesterol }\end{array}$ & 1.00 & $1.48(0.81-2.71)$ & $1.77(0.95-3.29)$ & $1.93(0.87-4.29)$ \\
\hline $\begin{array}{l}\text { Reduced } \\
\text { HDL- } \\
\text { cholesterol }\end{array}$ & 1.00 & $1.54(1.07-2.22)$ & $1.53(1.08-2.18)$ & $2.66(1.62-4.36)$ \\
\hline $\begin{array}{l}\text { Metabolic } \\
\text { syndrome }\end{array}$ & 1.00 & $1.51(1.02-2.22)$ & $1.73(1.16-2.59)$ & $2.42(1.41-4.15)$ \\
\hline $\begin{array}{l}\text { Increased } \\
\text { waist } \\
\text { circumference }\end{array}$ & 1.00 & $1.14(0.84-1.55)$ & $0.96(0.71-1.30)$ & $1.21(0.77-1.92)$ \\
\hline $\begin{array}{l}\text { Elevated } \\
\text { blood } \\
\text { pressure }\end{array}$ & 1.00 & $0.85(0.64-1.13)$ & $1.04(0.77-1.39)$ & $0.76(0.49-1.17)$ \\
\hline $\begin{array}{l}\text { Reduced } \\
\text { HDL- } \\
\text { cholesterol }\end{array}$ & 1.00 & $1.54(1.07-2.22)$ & $1.53(1.08-2.18)$ & $2.66(1.62-4.36)$ \\
\hline $\begin{array}{l}\text { Elevated } \\
\text { triglycerides }\end{array}$ & 1.00 & $1.31(0.99-1.75)$ & $1.41(1.04-1.91)$ & $1.34(0.86-2.08)$ \\
\hline $\begin{array}{l}\text { Elevated } \\
\text { fasting } \\
\text { glucose }\end{array}$ & 1.00 & $1.26(0.90-1.75)$ & $1.41(1.02-1.95)$ & $1.37(0.86-2.18)$ \\
\hline Hypertension & 1.00 & $1.02(0.68-1.52)$ & $1.25(0.83-1.89)$ & $0.86(0.49-1.53)$ \\
\hline Type 2 diabetes & 1.00 & $0.93(0.40-2.20)$ & $1.34(0.61-2.95)$ & $1.47(0.51-4.21)$ \\
\hline Women & MCP $(n=589)$ & MCA $(n=1217)$ & $\mathrm{HCP}(n=2673)$ & HCA $(n=290)$ \\
\hline \multicolumn{5}{|l|}{ Dyslipidemia } \\
\hline $\begin{array}{l}\text { Elevated total } \\
\text { cholesterol }\end{array}$ & 1.00 & $1.25(0.79-1.99)$ & $1.38(0.88-2.17)$ & $1.51(0.80-2.86)$ \\
\hline $\begin{array}{l}\text { Elevated } \\
\text { triglycerides }\end{array}$ & 1.00 & $1.15(0.69-1.91)$ & $1.00(0.63-1.60)$ & $1.50(0.74-3.01)$ \\
\hline $\begin{array}{l}\text { Elevated LDL- } \\
\text { cholesterol }\end{array}$ & 1.00 & $1.08(0.67-1.74)$ & $1.20(0.76-1.89)$ & $1.04(0.52-2.08)$ \\
\hline $\begin{array}{l}\text { Reduced } \\
\text { HDL- } \\
\text { cholesterol }\end{array}$ & 1.00 & $1.05(0.66-1.68)$ & $1.28(0.82-2.00)$ & $0.88(0.46-1.68)$ \\
\hline $\begin{array}{l}\text { Metabolic } \\
\text { syndrome }\end{array}$ & 1.00 & $0.97(0.61-1.54)$ & $1.06(0.69-1.62)$ & $1.14(0.64-2.06)$ \\
\hline $\begin{array}{l}\text { Increased } \\
\text { waist } \\
\text { circumference }\end{array}$ & 1.00 & $0.98(0.70-1.37)$ & $1.03(0.77-1.39)$ & $0.76(0.48-1.23)$ \\
\hline $\begin{array}{l}\text { Elevated } \\
\text { blood } \\
\text { pressure }\end{array}$ & 1.00 & $1.24(0.85-1.83)$ & $1.10(0.76-1.57)$ & $1.40(0.88-2.22)$ \\
\hline $\begin{array}{l}\text { Reduced } \\
\text { HDL- } \\
\text { cholesterol }\end{array}$ & 1.00 & $1.12(0.89-1.43)$ & $1.30(1.03-1.65)$ & $1.01(0.70-1.46)$ \\
\hline $\begin{array}{l}\text { Elevated } \\
\text { triglycerides }\end{array}$ & 1.00 & $0.87(0.61-1.23)$ & $0.89(0.64-1.23)$ & $1.02(0.64-1.63)$ \\
\hline
\end{tabular}


Table 5 Multivariable-adjusted odds ratios and 95\% confidence intervals of cardiovascular risk factors according to moderate- and high-carbohydrate diets, stratified by protein source (Continued)

\begin{tabular}{llccc}
$\begin{array}{l}\text { Elevated } \\
\text { fasting } \\
\text { glucose }\end{array}$ & 1.00 & $1.33(0.97-1.83)$ & $1.22(0.90-1.64)$ & $1.20(0.77-1.85)$ \\
$\begin{array}{l}\text { Hypertension } \\
\text { Type 2 diabetes }\end{array}$ & 1.00 & $1.23(0.69-2.22)$ & $1.21(0.72-2.03)$ & $1.60(0.79-3.25)$ \\
\hline
\end{tabular}

Abbreviations: HDL High-density lipoprotein-cholesterol, $L D L$ Low-density lipoprotein-cholesterol

${ }^{a} \mathrm{MCP}=$ carbohydrate intake $50-60 \%$ of energy + plant/animal protein $\geq 1 ; \mathrm{MCA}=$ carbohydrate intake $50-60 \%$ of energy + plant/animal protein $<1 ; \mathrm{HCP}=$ carbohydrate intake $\geq 70 \%$ of energy + plant/animal protein $\geq 1 ; \mathrm{HCA}=$ carbohydrate intake $\geq 70 \%$ of energy + plant/animal protein $<1$

${ }^{\mathrm{b}}$ Adjusted for age, body mass index (except the model of waist circumference), education, household income, physical activity, smoking, survey period, alcohol consumption, and total energy intake

increased ORs for an elevated triglyceride level (OR, 1.41; 95\% CI, 1.04-1.91) and an elevated fasting glucose level (OR, 1.41; 95\% CI, 1.02-1.95), compared with MCP. Men in the moderate-carbohydrate diet with animal protein (MCA) group had higher ORs for an elevated total cholesterol level (OR, 1.80; 95\% CI, 1.173.04), reduced HDL-cholesterol level (OR, 1.54; 95\% CI, $1.07-2.22)$, and metabolic syndrome (OR, 1.51; $95 \% \mathrm{CI}$, $1.02-2.22)$ than men in the MCP group. In women, only the HCP group had an increased OR for a reduced HDL-cholesterol level (OR, 1.30; 95\% CI, 1.03-1.65), compared with the MCP group.

Considering the age differences among participants on the four types of diets, an age-stratified analysis was conducted for each sex (Table 6, Supplementary Table 1). In younger adults aged 19-49 years, most significant associations were maintained in men and women, except for elevated total cholesterol in men with an HCA (Table 6). Younger women with an MCA (OR, 2.21; 95\% CI, 1.11-4.38) and those with an HCP (OR, 2.38; 95\% CI, 1.20-4.73) also had increased ORs for an elevated total cholesterol level, compared with those with an MCP. Younger women who had an HCP also exhibited a higher OR for an elevated fasting glucose level (OR, 1.57; 95\% CI, 1.072.29 ), compared with younger women who had an MCP. In contrast, among adults aged $\geq 50$ years, no significant associations were found in men, whereas several cardiovascular risk factors were associated with diets in women (Supplementary Table 1). Compared with the MCP group, the MCA group had higher ORs for metabolic syndrome including reduced HDL-cholesterol; the HCP and HCA groups had higher ORs for hypertension in older women.

\section{Discussion}

This large-scale cross-sectional study of Korean adults compared associations of cardiovascular risk factors with moderate- and high-carbohydrate diets according to protein source. It showed that a moderate-carbohydrate diet with plant protein (MCP) was inversely associated with several cardiovascular risk factors, including elevated total cholesterol, reduced HDL-cholesterol, and elevated fasting glucose, especially in younger men and women.

Early studies reported inconsistent associations of dietary carbohydrate with cardiovascular risk factors between Asian and Western populations [26-31], which might be attributable to different ranges of carbohydrate intakes between these populations. A recent crosssectional study reported that Korean adults consume more carbohydrates than US adults; a stronger association of dietary carbohydrate with metabolic syndrome was found in Korean adults [32]. Seidelmann et al. [12] reported a U-shaped association of carbohydrate intake with mortality, whereby Asian populations represented the right side of the curve and North American and European populations the left side. Therefore, nutritional targets for preventing cardiovascular risk factors should be differentiated by considering different ranges of carbohydrate intake that are typical of Asian and Western countries. For Asian populations whose diets are typically high in carbohydrate, it is important to reduce the carbohydrate intake to a moderate level.

To lower carbohydrate intake, both protein and fat intakes can be increased without restriction or protein intake can be increased while allowing fat intake to reach an appropriate level [11]. Substitution of carbohydrates for dietary protein reduces the postprandial glycemic response [33, 34]. Although associations between dietary protein and cardiovascular risks have not been investigated in detail, a study of Asian Indians reported that a high-protein diet (29\% of energy from protein) for 3 months promoted weight loss, compared with a standard diet (15\% of energy from protein) in overweight and obese adults [35].

In this study, participants with a moderate carbohydrate diet with animal protein (MCA) had higher ORs for lipid abnormalities and metabolic syndrome compared with those with a moderate carbohydrate diet with plant protein (MCP), except among older adult men. These findings were consistent with the results of previous prospective cohort studies, which reported differences in associations of cardiovascular risks with animal- or plant-based low-carbohydrate diets in 
Table 6 Multivariable-adjusted odds ratios and 95\% confidence intervals of cardiovascular risk factors according to moderate- and high-carbohydrate diets, stratified by protein source in younger adults (aged 19-49 years)

\begin{tabular}{|c|c|c|c|c|}
\hline Men & $\begin{array}{l}\text { Moderate carbohydrate diet } \\
\text { with plant protein (MCP) } \\
(n=295)\end{array}$ & $\begin{array}{l}\text { Moderate carbohydrate diet with } \\
\text { animal protein }(M C A)^{\mathrm{a}}(n=774)\end{array}$ & $\begin{array}{l}\text { High carbohydrate diet } \\
\text { with plant protein }(\mathrm{HCP})^{\mathrm{a}} \\
(n=606)\end{array}$ & $\begin{array}{l}\text { High carbohydrate diet } \\
\text { with animal protein } \\
(H C A)^{a} \\
(n=112)\end{array}$ \\
\hline \multicolumn{5}{|l|}{ Dyslipidemia } \\
\hline $\begin{array}{l}\text { Elevated total } \\
\text { cholesterol }\end{array}$ & $1.00^{\mathrm{b}}$ & $2.22(1.16-4.24)$ & $2.57(1.34-4.91)$ & $2.56(1.00-6.56)$ \\
\hline $\begin{array}{l}\text { Elevated } \\
\text { triglycerides }\end{array}$ & 1.00 & $1.04(0.70-1.55)$ & $1.10(0.72-1.67)$ & $1.00(0.50-2.02)$ \\
\hline $\begin{array}{l}\text { Elevated LDL- } \\
\text { cholesterol }\end{array}$ & 1.00 & $2.20(0.96-5.04)$ & $3.02(1.33-6.87)$ & $2.55(0.82-7.97)$ \\
\hline $\begin{array}{l}\text { Reduced } \\
\text { HDL- } \\
\text { cholesterol }\end{array}$ & 1.00 & $1.75(1.15-2.67)$ & $1.92(1.26-2.92)$ & $3.69(1.98-6.89)$ \\
\hline $\begin{array}{l}\text { Metabolic } \\
\text { syndrome }\end{array}$ & 1.00 & $2.07(1.29-3.32)$ & $2.97(1.76-5.03)$ & $3.96(1.87-8.40)$ \\
\hline $\begin{array}{l}\text { Increased } \\
\text { waist } \\
\text { circumference }\end{array}$ & 1.00 & $1.13(0.80-1.60)$ & $0.91(0.64-1.31)$ & $1.34(0.77-2.33)$ \\
\hline $\begin{array}{l}\text { Elevated } \\
\text { blood } \\
\text { pressure }\end{array}$ & 1.00 & $0.84(0.60-1.19)$ & $1.29(0.89-1.86)$ & $0.81(0.45-1.45)$ \\
\hline $\begin{array}{l}\text { Reduced } \\
\text { HDL- } \\
\text { cholesterol }\end{array}$ & 1.00 & $1.75(1.15-2.67)$ & $1.92(1.26-2.92)$ & $3.69(1.98-6.89)$ \\
\hline $\begin{array}{l}\text { Elevated } \\
\text { triglycerides }\end{array}$ & 1.00 & $1.42(1.02-1.98)$ & $1.61(1.12-2.31)$ & $1.46(0.83-2.57)$ \\
\hline $\begin{array}{l}\text { Elevated } \\
\text { fasting } \\
\text { glucose }\end{array}$ & 1.00 & $1.35(0.89-2.06)$ & $2.06(1.34-3.18)$ & $1.94(1.02-3.69)$ \\
\hline Hypertension & 1.00 & $1.01(0.63-1.64)$ & $1.72(1.03-2.87)$ & $1.01(0.45-2.25)$ \\
\hline Women & $\operatorname{MCP}(n=452)$ & MCA $(n=988)$ & $\mathrm{HCP}(n=1163)$ & HCA $(n=174)$ \\
\hline \multicolumn{5}{|l|}{ Dyslipidemia } \\
\hline $\begin{array}{l}\text { Elevated total } \\
\text { cholesterol }\end{array}$ & 1.00 & $2.21(1.11-4.38)$ & $2.38(1.20-4.73)$ & $2.02(0.75-5.41)$ \\
\hline $\begin{array}{l}\text { Elevated } \\
\text { triglycerides }\end{array}$ & 1.00 & $0.94(0.50-1.76)$ & $0.88(0.47-1.66)$ & $1.18(0.47-2.98)$ \\
\hline $\begin{array}{l}\text { Elevated LDL- } \\
\text { cholesterol }\end{array}$ & 1.00 & $1.76(0.84-3.70)$ & $2.07(0.99-4.31)$ & $1.08(0.31-3.78)$ \\
\hline $\begin{array}{l}\text { Reduced } \\
\mathrm{HDL}- \\
\text { cholesterol }\end{array}$ & 1.00 & $1.07(0.62-1.86)$ & $1.52(0.89-2.61)$ & $1.34(0.64-2.79)$ \\
\hline $\begin{array}{l}\text { Metabolic } \\
\text { syndrome }\end{array}$ & 1.00 & $0.69(0.36-1.29)$ & $0.95(0.51-1.77)$ & $0.81(0.36-1.81)$ \\
\hline $\begin{array}{l}\text { Increased } \\
\text { waist } \\
\text { circumference }\end{array}$ & 1.00 & $0.98(0.66-1.45)$ & $1.09(0.76-1.58)$ & $0.88(0.47-1.67)$ \\
\hline $\begin{array}{l}\text { Elevated } \\
\text { blood } \\
\text { pressure }\end{array}$ & 1.00 & $1.36(0.81-2.27)$ & $1.14(0.67-1.96)$ & $1.68(0.90-3.17)$ \\
\hline $\begin{array}{l}\text { Reduced } \\
\text { HDL- } \\
\text { cholesterol }\end{array}$ & 1.00 & $1.01(0.77-1.34)$ & $1.37(1.03-1.81)$ & $0.94(0.60-1.48)$ \\
\hline $\begin{array}{l}\text { Elevated } \\
\text { trialycerides }\end{array}$ & 1.00 & $0.76(0.49-1.17)$ & $0.85(0.55-1.31)$ & $0.88(0.47-1.66)$ \\
\hline
\end{tabular}


Table 6 Multivariable-adjusted odds ratios and 95\% confidence intervals of cardiovascular risk factors according to moderate- and high-carbohydrate diets, stratified by protein source in younger adults (aged 19-49 years) (Continued)

\begin{tabular}{|c|c|c|c|c|}
\hline $\begin{array}{l}\text { Elevated } \\
\text { fasting } \\
\text { glucose }\end{array}$ & 1.00 & $1.29(0.87-1.91)$ & $1.57(1.07-2.29)$ & $1.26(0.70-2.27)$ \\
\hline Hypertension & 1.00 & $1.23(0.56-2.72)$ & $0.92(0.41-2.09)$ & $1.36(0.44-4.21)$ \\
\hline
\end{tabular}

Abbreviations: HDL High-density lipoprotein-cholesterol, LDL Low-density lipoprotein-cholesterol

${ }^{\mathrm{a}} \mathrm{MCP}=$ carbohydrate intake $50-60 \%$ of energy + plant/animal protein $\geq 1 ; \mathrm{MCA}=$ carbohydrate intake $50-60 \%$ of energy + plant/animal protein $<1 ; \mathrm{HCP}=$ carbohydrate intake $\geq 70 \%$ of energy + plant/animal protein $\geq 1 ; \mathrm{HCA}=$ carbohydrate intake $\geq 70 \%$ of energy + plant/animal protein $<1$

${ }^{b}$ Adjusted for body mass index (except the model of waist circumference), education, household income, physical activity, smoking, survey period, alcohol consumption, and total energy intake

American adults [36-39]. Plant protein, especially soy protein, and the intake of isoflavone reportedly reduced the total and LDL-cholesterol levels compared with animal protein [16]; moreover, they showed beneficial associations with inflammation and oxidative stress [40].

Few studies of dietary pattern or soy protein intake in Koreans have been conducted. A dietary pattern including high intake of whole grains and beans was associated with a lower risk of insulin resistance in Korean adults [41]. Additionally, a prospective cohort study reported that soy protein and isoflavone intake were related to a reduced risk of metabolic syndrome among middle-aged Korean adults [42].

Further studies are needed to optimize protein intake along with food source in moderate-carbohydrate diets in Koreans. The findings of this study indicate that adequate intake of plant protein with a moderate carbohydrate intake $(50-60 \%$ of energy) may ameliorate metabolic diseases. Further studies are warranted to confirm the effects of this type of diet on cardiovascular risk factors in Koreans.

This study had several limitations. First, its crosssectional design prevented investigation of the causal relationship of a moderate- or high-carbohydrate diet with cardiovascular risk factors according to source of protein. Further clinical trials are needed to verify the effects of a moderate-carbohydrate diet with plant protein (MCP) on cardiovascular risk factors in Koreans. Second, dietary intake was measured using 1-day 24-h dietary recall, which might not represent the usual intake of participants. Third, participants were grouped based on their dietary intake, which caused the number of participants to differ among the groups. Thus, caution is required when interpreting the results, especially for older adults. Fourth, the study did not consider fat intake. However, a previous study defined a low-carbohydrate diet using all macronutrients; it showed that a lowcarbohydrate diet was not associated with metabolic diseases, with the exception of a reduced HDL-cholesterol level, in Korean adults [43]. We propose that macronutrient composition is evaluated in various ways to develop effective dietary recommendations for prevention of cardiovascular diseases. Despite these limitations, to our knowledge, this study is the first to investigate associations of moderate- and high-carbohydrate diets with cardiovascular risk factors considering protein intake among Korean adults. Moreover, the finding that an MCP was inversely associated with cardiovascular risk factors was novel.

\section{Conclusions}

A moderate-carbohydrate diet with a high intake of plant protein was inversely associated with cardiovascular risk factors, especially among younger Korean adults. Further intervention studies that consider protein sources and carbohydrate intake levels are required to develop a diet that enhances the cardiovascular health of Koreans.

\section{Supplementary information}

Supplementary information accompanies this paper at https://doi.org/10. 1186/s12937-020-00603-2.

Additional file 1: Supplementary Table 1. Multivariable-adjusted odds ratios and $95 \%$ confidence intervals of cardiovascular risk factors according to moderate- and high-carbohydrate diets, stratified by protein source in older adults (aged $\geq 50$ years).

\section{Abbreviations}

BMI: Body mass index; Cl: Confidence interval; DBP: Diastolic blood pressure; EER: Estimated energy requirement; HCA: High-carbohydrate diet with animal protein; HCP: High-carbohydrate diet with plant protein; HDL: High-density lipoprotein-cholesterol; KCDC: Korea Centers for Disease Control \& Prevention; KDRI: Dietary Reference Intakes for Koreans; KNHANES: Korea National Health and Nutrition Examination Survey; LDL: Low-density lipoprotein-cholesterol; MCA: Moderate-carbohydrate diet with animal protein; MCP: Moderate-carbohydrate diet with plant protein; MFEB: Meat, fish, eggs, and beans; OR: Odds ratio; SBP: Systolic blood pressure; SE: Standard error; US: United States

\section{Acknowledgements}

Not applicable.

\section{Authors' contributions}

$\mathrm{KH}$ analyzed the data and wrote the draft manuscript. KN critically revised the manuscript and commented on the manuscript. YS supervised the work and had the primary responsibility for the final content. All authors read and approved the manuscript.

\section{Funding}

This work was supported by the 2019 research fund, of the Catholic University of Korea and a research grant from Pulmuone Co., Ltd. 


\section{Availability of data and materials}

The datasets used and/or analyzed during the current study are available in https://knhanes.cdc.go.kr/knhanes/eng/index.do.

\section{Ethics approval and consent to participate}

The survey protocol of KNHANES was approved by the KCDC Institutional Review Board until 2014, and has been conducted without deliberation according to the bioethics law since 2015 (2013-07CON-03-4C and 201312EXP-03-5C). Informed consent was obtained from all participants.

\section{Consent for publication}

Not applicable.

\section{Competing interests}

All authors declare no potential conflict of interest.

\section{Author details}

'Graduate School of Public Health, Seoul National University, Seoul 08826, Korea. ${ }^{2}$ Health \& Nutrition Research Center, Pulmuone Co., Ltd., Seoul 06367, Korea. ${ }^{3}$ Major of Food and Nutrition, The Catholic University of Korea, 43 Jibong-ro, Wonmi-gu, Bucheon-si, Gyeonggi-do 14662, Republic of Korea.

Received: 28 January 2020 Accepted: 10 August 2020

Published online: 14 August 2020

\section{References}

1. Astrup A, Grunwald GK, Melanson EL, Saris WH, Hill JO. The role of low-fat diets in body weight control: a meta-analysis of ad libitum dietary intervention studies. Int J Obes Relat Metab Disord. 2000;24(12):1545-52.

2. Astrup A, Meinert Larsen T, Harper A. Atkins and other lowcarbohydrate diets: hoax or an effective tool for weight loss? Lancet. 2004;364(9437):897-9.

3. Hu T, Mills KT, Yao L, Demanelis K, Eloustaz M, Yancy WS Jr, Kelly TN, He J, Bazzano LA. Effects of low-carbohydrate diets versus low-fat diets on metabolic risk factors: a meta-analysis of randomized controlled clinical trials. Am J Epidemiol. 2012;176(Suppl 7):S44-54.

4. Mansoor N, Vinknes KJ, Veierod MB, Retterstøl K. Effects of low-carbohydrate diets $\mathrm{v}$. low-fat diets on body weight and cardiovascular risk factors: a metaanalysis of randomised controlled trials. Br J Nutr. 2016;115(3):466-79.

5. Wylie-Rosett J, Aebersold K, Conlon B, Isasi CR, Ostrovsky NW. Health effects of low-carbohydrate diets: where should new research go? Curr Diab Rep. 2013;13(2):271-8.

6. U.S. Department of Agriculture, Agricultural Research Service. Energy intakes: percentages of energy from protein, carbohydrate, fat, and alcohol, by gender and age, what we eat in America, NHANES 2015-2016. Beltsville: Agricultural Research Service; 2018.

7. Public Health England. National diet and nutrition survey: UK results from years 7 and 8 (combined) of the rolling programme (2014/15-2015/2016). London: Public Health England; 2018.

8. Statistics Canada. Health fact sheets: nutrient intakes from food, 2015. 2017. https://www150.statcan.gc.ca/n1/pub/82-625-x/2017001/article/14830-eng. htm. Accessed Apr 2018.

9. Ministry of Health and Welfare, Korea Centers for Disease Control and Prevention. Korea health statistics 2017: Korea National Health and Nutrition Examination Survey (KNHANES VII-2). Sejong: Ministry of Health and Welfare; 2018.

10. Ministry of Health, Labour and Welfare. The national health and nutrition survey in Japan, 2016. Tokyo: Ministry of Health, Labour and Welfare; 2018.

11. Naude CE, Schoonees A, Senekal M, Young T, Garner P, Volmink J. Low carbohydrate versus isoenergetic balanced diets for reducing weight and cardiovascular risk: a systematic review and meta-analysis. PLoS One. 2014; 9(7):e100652.

12. Seidelmann SB, Claggett B, Cheng S, Henglin M, Shah A, Steffen LM, Folsom AR, Rimm EB, Willett WC, Solomon SD. Dietary carbohydrate intake and mortality: a prospective cohort study and meta-analysis. Lancet Public Health. 2018;3(9):e419-28.

13. Noakes M, Keogh JB, Foster PR, Clifton PM. Effect of an energy-restricted, high-protein, low-fat diet relative to a conventional high-carbohydrate, lowfat diet on weight loss, body composition, nutritional status, and markers of cardiovascular health in obese women. Am J Clin Nutr. 2005;81(6):1298-306.
14. Morenga LT, Williams S, Brown R, Mann J. Effect of a relatively high-protein, high-fiber diet on body composition and metabolic risk factors in overweight women. Eur J Clin Nutr. 2010;64(11):1323-31.

15. Richter CK, Skulas-Ray AC, Champagne CM, Kris-Etherton PM. Plant protein and animal proteins: do they differentially affect cardiovascular disease risk? Adv Nutr. 2015;6(6):712-28.

16. Chalvon-Demersay T, Azzout-Marniche D, Arfsten J, Egli L, Gaudichon C, Karagounis $L G$, Tomé D. A systematic review of the effects of plant compared with animal protein sources on features of metabolic syndrome. J Nutr. 2017;147(3):281-92.

17. Tharrey M, Mariotti F, Mashchak A, Barbillon P, Delattre M, Fraser GE. Patterns of plant and animal protein intake are strongly associated with cardiovascular mortality: the Adventist Health Study-2 cohort. Int J Epidemiol. 2018;47(5):1603-12.

18. Shang X, Scott D, Hodge A, English DR, Giles GG, Ebeling PR, Sanders KM Dietary protein from different food sources, incident metabolic syndrome and changes in its components: an 11-year longitudinal study in healthy community-dwelling adults. Clin Nutr. 2017;36(6):1540-8.

19. Kweon S, Kim Y, Jang MJ, Kim Y, Kim K, Choi S, Chun C, Khang YH, Oh K. Data resource profile: the Korea National Health and Nutrition Examination Survey (KNHANES). Int J Epidemiol. 2014;43(1):69-77.

20. Ministry of Health and Welfare, The Korean Nutrition Society. Dietary reference intakes for Koreans 2015. Seoul: The Korean Nutrition Society; 2015.

21. Freeman M. In: Runge M, Patterson C, editors. Lipid metabolism and coronary artery disease. Totowa: Humana Press; 2006.

22. Ministry of Health and Welfare. Korea Centers for Disease Control and Prevention. Manual for the sixth (2013-2015) Korea national health and nutrition examination survey: health examination. Osong: Korea Centers for Disease Control and Prevention; 2014.

23. The Korean Society of Lipid and Atherosclerosis. Korean guidelines for the management of dyslipidemia 4th ed. Seoul: The Korean Society of Lipid and Atherosclerosis; 2018.

24. Grundy SM, Cleeman JI, Daniels SR, Donato KA, Eckel RH, Franklin BA, Gordon DJ, Krauss RM, Savage PJ, Smith SC Jr, Spertus JA, Costa F. Diagnosis and management of the metabolic syndrome: an American heart association/national heart, lung, and blood institute scientific statement. Circulation. 2005;112(17):2735-52.

25. Lee SY, Park HS, Kim DJ, Han JH, Kim SM, Cho GJ, Kim DY, Kwon HS, Kim SR, Lee $\mathrm{CB}$, Oh SJ, Park CY, Yoo HJ. Appropriate waist circumference cutoff points for central obesity in Korean adults. Diabetes Res Clin Pract. 2007; 75(1):72-80

26. Ha K, Joung H, Song $Y$. Inadequate fat or carbohydrate intake was associated with an increased incidence of type 2 diabetes mellitus in Korean adults: a 12-year community-based prospective cohort study. Diabetes Res Clin Pract. 2019;148:254-61.

27. Nanri A, Mizoue T, Noda M, Takahashi Y, Kato M, Inoue M, Tsugane S. Rice intake and type 2 diabetes in Japanese men and women: the Japan public health center-based prospective study. Am J Clin Nutr. 2010;92(6):1468-77.

28. Villegas R, Liu S, Gao YT, Yang G, Li H, Zheng W, Shu XO. Prospective study of dietary carbohydrates, glycemic index, glycemic load, and incidence of type 2 diabetes mellitus in middle-aged Chinese women. Arch Intern Med. 2007;167(21):2310-6.

29. Hodge AM, English DR, O'Dea K, Giles GG. Glycemic index and dietary fiber and the risk of type 2 diabetes. Diabetes Care. 2004;27(11):2701-6.

30. Schulze MB, Schulz M, Heidemann C, Schienkiewitz A, Hoffmann K, Boeing $\mathrm{H}$. Carbohydrate intake and incidence of type 2 diabetes in the European Prospective Investigation into Cancer and Nutrition (EPIC)-Potsdam Study. Br J Nutr. 2008;99(5):1107-16.

31. AlEssa HB, Bhupathiraju SN, Malik VS, Wedick NM, Campos H, Rosner B, Willett WC, Hu FB. Carbohydrate quality and quantity and risk of type 2 diabetes in US women. Am J Clin Nutr. 2015;102(6):1543-53.

32. Ha K, Kim K, Chun OK, Joung H, Song Y. Differential association of dietary carbohydrate intake with metabolic syndrome in the US and Korean adults: data from the 2007-2012 NHANES and KNHANES. Eur J Clin Nutr. 2018;72(6):848-60.

33. Pi-Sunyer FX. Glycemic index and disease. Am J Clin Nutr. 2002;76(1): 290S-8S.

34. Meng $H$, Matthan NR, Ausman LM, Lichtenstein AH. Effect of macronutrients and fiber on postprandial glycemic responses and meal glycemic index and glycemic load value determinations. Am J Clin Nutr. 2017;105(4):842-53. 
35. Gulati S, Misra A, Tiwari R, Sharma M, Pandey RM, Yadav CP. Effect of high-protein meal replacement on weight and cardiometabolic profile in overweight/obese Asian Indians in North India. Br J Nutr. 2017; 117(11):1531-40.

36. Halton TL, Willett WC, Liu S, Manson JE, Albert CM, Rexrode K, Hu FB. Lowcarbohydrate-diet score and the risk of coronary heart disease in women. N Engl J Med. 2006;355(19):1991-2002.

37. Halton TL, Liu S, Manson JE, Hu FB. Low-carbohydrate-diet score and risk of type 2 diabetes in women. Am J Clin Nutr. 2008;87(2):339-46.

38. de Koning L, Fung TT, Liao X, Chiuve SE, Rimm EB, Willett WC, Spiegelman D, Hu FB. Low-carbohydrate diet scores and risk of type 2 diabetes in men. Am J Clin Nutr. 2011;93(4):844-50.

39. Bao W, Li S, Chavarro JE, Tobias DK, Zhu Y, Hu FB, Zhang C. Low carbohydrate-diet scores and long-term risk of type 2 diabetes among women with a history of gestational diabetes mellitus: a prospective cohort study. Diabetes Care. 2016;39(1):43-9.

40. Hruby A, Jacques PF. Dietary protein and changes in biomarkers of inflammation and oxidative stress in the Framingham Heart Study Offspring Cohort. Curr Dev Nutr. 2019;3(5):nzz019.

41. Song S, Paik HY, Song Y. High intake of whole grains and beans pattern is inversely associated with insulin resistance in healthy Korean adult population. Diabetes Res Clin Pract. 2012;98(3):e28-31.

42. Woo HW, Kim MK, Lee YH, Shin DH, Shin MH, Choi BY. Habitual consumption of soy protein and isoflavones and risk of metabolic syndrome in adults $\geq 40$ years old: a prospective analysis of the Korean multi-rural communities cohort study (MRCohort). Eur J Nutr. 2019;58(7): 2835-50.

43. Ha K, Joung H, Song Y. Low-carbohydrate diet and the risk of metabolic syndrome in Korean adults. Nutr Metab Cardiovasc Dis. 2018;28(11):1122-32.

\section{Publisher's Note}

Springer Nature remains neutral with regard to jurisdictional claims in published maps and institutional affiliations.

Ready to submit your research? Choose BMC and benefit from:

- fast, convenient online submission

- thorough peer review by experienced researchers in your field

- rapid publication on acceptance

- support for research data, including large and complex data types

- gold Open Access which fosters wider collaboration and increased citations

- maximum visibility for your research: over $100 \mathrm{M}$ website views per year

At $\mathrm{BMC}$, research is always in progress.

Learn more biomedcentral.com/submissions 